\title{
Invariant Natural Killer T Cells Promote T Cell Immunity by Modulating the Function of Lung Dendritic Cells during Chlamydia pneumoniae Infection
}

\author{
Sudhanshu Shekhar ${ }^{\mathrm{a}}$ Antony George Joyee ${ }^{\mathrm{b}} \quad$ Xiaoling Gao $^{\mathrm{a}} \quad$ Ying Peng $^{\mathrm{a}}$ \\ Shuhe Wang ${ }^{\mathrm{a}}$ Jie Yang $^{\mathrm{a}}$ Xi Yang ${ }^{\mathrm{a}, \mathrm{b}}$ \\ a Laboratory for Infection and Immunity, Department of Medical Microbiology and ${ }^{\mathrm{b}}$ Department of Immunology, \\ Faculty of Medicine, University of Manitoba, Winnipeg, Man., Canada
}

\section{Key Words}

Invariant natural killer T cells · Lung dendritic cells · T cells .

Chlamydia pneumoniae

\begin{abstract}
In this study, we examined the effect of invariant natural killer T (iNKT) cells on the function of lung dendritic cells (LDCs) in eliciting protective immunity against Chlamydia pneumoniae (Cpn) lung infection. We employed a combination of approaches including the use of iNKT cell-deficient, Ja18knockout (KO) mice and LDC adoptive transfer. We found that iNKT cells significantly altered the number, phenotype and cytokine profile of LDCs following infection. Furthermore, coculture of T cells with LDCs from Cpn-infected wildtype (WT) and KO mice induced type- 1 and type- 2 responses, respectively. More importantly, upon adoptive transfer, LDCs from Cpn-infected WT mice (WT-LDCs) conferred protective immunity, whereas LDCs from KO mice (KO-LDCs) increased the severity of disease after challenge infection. Further cytokine analyses of the lung tissues and lung-draining lymph node cells showed that KO-LDC-recipient mice exhibited a type- 2 cytokine production pattern, while WT-LDC recipients exhibited a type-1 cytokine profile. Taken together, our results provide in vivo evidence that iNKT cells play a
\end{abstract}

critical role in modulating LDC function to generate protective T-cell immunity, particularly in a clinically relevant intracellular bacterial infection.

(c) 2014 S. Karger AG, Basel

\section{Introduction}

The lung is a vital organ that facilitates gaseous exchange during respiration. In doing so, the lung is continually exposed to a plethora of environmental pathogens, including bacteria and viruses. Lung dendritic cells (LDCs) are the chief orchestrators of immune responses in the lung because of their unique ability to process and present antigens in the most efficient way. LDCs express various surface molecules like MHC-II and costimulatory molecules, and are located within the epithelium and interstitium of the lung, functioning as a cellular interface between the external and internal lung environment [1]. They acquire microbial antigens and undergo a coordinated maturation program that enables them not only to upregulate MHC-II and costimulatory molecules and induce enhanced cytokine production, but also to migrate to the lung-draining mediastinal lymph nodes (MLNs) to prime naïve $\mathrm{T}$ cells to generate adaptive immune respons- 
es [2]. In the steady-state condition, LDCs produce cytokines such as IL- 6 and IL-10 and preferentially polarize T cells towards a type- 2 response $[3,4]$. During respiratory infections, LDCs can, however, generate different immune responses, depending upon the type of pathogen. For example, LDCs induce type-1 T-cell responses characterized by IFN- $\gamma$ production against intracellular bacteria and viruses, while exposure to extracellular pathogens such as parasites mounts type- $2 \mathrm{~T}$-cell responses that lead to eosinophilia and IgE antibody production [5-7].

Invariant natural killer T (iNKT) cells represent a subset of innate lymphocytes that possess the characteristics of both NK and $\alpha \beta$ T cells. iNKT cells express the $\alpha \beta$ T-cell receptor with the use of chain Va14-Ja18 in mice and Va24-Ja18 in humans. They also express markers of NK cells, such as NK1.1 [8]. Unlike conventional T cells, iNKT cells recognize glycolipid and lipid antigens presented to them by nonclassical MHC-I CD1d molecules. The prototypic antigen used in experimental studies for iNKT cell activation is $\alpha$-galactosylceramide ( $\alpha$-GalCer) which is, biochemically, a glycolipid derived from marine sponges. Upon stimulation with specific antigens, iNKT cells promptly produce a variety of cytokines, most notably IFN- $\gamma$ and IL-4 [9]. Many glycolipid and lipid antigens specific for the activation of iNKT cells have also been derived from several bacterial species [9]. Recently, we identified a glycolipid exoantigen, GLXA, from Chlamydia muridarum (a mouse biovar of $C$. trachomatis) that induced iNKT cells to express higher levels of an activation marker, CD69, and to produce cytokines like IFN- $\gamma$ and IL-4 [10]. These findings suggest a direct role of the invariant $\mathrm{T}$-cell receptor of iNKT cells in the induction of immune responses. In addition to the direct recognition of antigens, iNKT cells can be indirectly activated through innate or inflammatory cytokine-driven stimuli during microbial infections. This indirect activation is the dominant pathway that is mediated through the secretion of IL-12 by antigen-presenting cells (APCs) following Toll-like receptor activation [11]. iNKT cells have been shown to play a crucial role in tumour rejection, maintenance of tolerance and protective immunity against many pathogens [9]. They not only contribute to innate immune responses but also influence the outcome of adaptive immune responses. The major cellular mechanism by which iNKT cells manipulate adaptive immune responses is by modulating the function of DCs. Thus, these cells act as a connecting link between innate and adaptive immunity [8].

C. pneumoniae (Cpn), an obligate intracellular bacterial pathogen, causes various diseases in humans, thus posing a threat to public health worldwide. These diseases include bronchitis, sinusitis and community-acquired pneumonia [12]. Serologic studies have shown that most people have been infected with Cpn at least once during their lifetime [13]. Recently, Cpn has been implicated in the pathogenesis of atherosclerosis, Alzheimer's disease, multiple sclerosis, arthritis and asthma [14]. At present, no vaccine is available for Cpn diseases. Efforts to develop a safe and rational chlamydial vaccine have been hindered by the lack of adequate understanding of the protective and pathologic immune responses to $\mathrm{Cpn}$ infection. Emerging evidence suggests that type- $1 \mathrm{~T}$-cell responses play a pivotal role in the clearance of $\mathrm{Cpn}$ infection, while type- 2 responses may result in immunopathology [14]. In addition, unlike $C$. trachomatis infection, CD8 T-cell responses, relative to $\mathrm{CD} 4 \mathrm{~T}$-cell responses, play a dominant role in Cpn infection.

We previously reported a significant modulating effect of iNKT cells on the function of DCs in the spleen (SDCs) to induce protective immunity against Cpn lung infection $[15,16]$. How iNKT cells interact with LDCs, which reside at the primary site of $\mathrm{Cpn}$ infection, i.e. the lung, where maximal inflammatory and pathologic changes occur, is an important question yet to be addressed. The question is further emphasized by emerging evidence that LDCs and SDCs reflect phenotypic and functional distinctions, which may be attributed to the influence of their local tissue environment $[3,4,17]$. In addition, functional differences between LDCs and SDCs have been reported in various infection models [18-20]. Recent studies further demonstrate that iNKT cells interact with LDCs in the lung microenvironment to induce local immune responses during respiratory infections $[21,22]$. Taking into consideration the facts that (1) phenotypic and functional distinctions between LDCs and SDCs could differentially contribute to the outcome of T cell responses, (2) iNKT cells elicit immune responses to respiratory pathogens by interacting with local DCs and (3) the events occurring at the secondary lymphoid organs such as the spleen may not necessarily mirror what happens in the primary organ of infection (the lung), we directly examined the influence of iNKT cells on the functional competence of LDCs in inducing protective immune responses against $\mathrm{Cpn}$ infection. We found that LDCs from Ja18-knockout (KO) mice, which lack iNKT cells, showed reduced expansion, lower MHC-II and costimulatory molecule expression and decreased IL-12 production when compared with those from wild-type (WT) mice post-infection (p.i.). Furthermore, LDCs from Cpn-infected $\mathrm{KO}$ mice (KO-LDCs) were less effi- 
cient in directing antigen-specific type-1 responses than LDCs from WT mice (WT-LDCs); this is supported by coculture data. Moreover, upon adoptive transfer, KOLDCs enhanced type- $2 \mathrm{~T}$ cell responses and increased the severity of Cpn infection, in sharp contrast to the effect with the transfer of WT-LDCs that promoted protective type-1 immunity against Cpn challenge. Together, our findings have shed light on the role played by iNKT cells in modulating the function of LDCs for generating T-cell immunity against chlamydial infection. To our knowledge, this is the first report demonstrating the effect of iNKT cells on LDC function in the lung microenvironment during a clinically relevant intracellular bacterial infection.

\section{Materials and Methods}

\section{Organism}

The propagation and infectivity determination of Cpn (AR-39 strain) were performed as described previously [15]. In brief, Cpninfected HeLa 229 cell monolayers were ultrasonically disrupted and the cell debris was removed by centrifugation. The bacteria were concentrated by high-speed centrifugation, resuspended in sucrose-phosphate-glutamic acid buffer and stored in small aliquots frozen at $-80^{\circ} \mathrm{C}$ until used. The same seed stock was used throughout the study. The infectivity, as measured by inclusionforming units (IFUs) of the bacterial preparation, was determined in HeLa cell culture. Highly purified Cpn elementary body (EB) preparations were obtained by renografin gradient separation. For in vitro antigenic restimulation assays and cytokine induction in culture, a UV-killed preparation of EB (UK-EB) was used. For infection, mice were mildly sedated with isoflurane and intranasally inoculated with $3 \times 10^{6}$ IFUs of Cpn in $40 \mu$ of PBS.

\section{Mice}

Ja18-KO and WT C57BL/6 mice were bred and kept at the pathogen-free animal care facility of the University of Manitoba. Breeding pairs of the homozygous Ja18-KO mice in C57BL/6 background were kindly provided by Dr. Masaru Taniguchi (RIKEN Research Center for Allergy and Immunology, Yokohama, Japan). The Ja18-KO mice were originally generated by specific deletion of the Ja18 gene segment using homologous recombination and aggregation chimera techniques. All mice used in this study were males of 6-8 weeks of age. All experiments were done in compliance with the guidelines issued by the Canadian Council of Animal Care, and the animal protocol was approved by the institutional ethics committee.

\section{LDC Isolation}

Lungs were harvested from the mice at day 3 after infection and then processed into single-cell suspensions. Briefly, the lungs were digested in $10 \mathrm{mg} / \mathrm{ml}$ collagenase XI (Roche Diagnostics, Meylan, France) in RPMI 1640 for $1 \mathrm{~h}$ at $37^{\circ} \mathrm{C}$. EDTA at $5 \mathrm{mM}$ was added during the last 5 min to disrupt the LDC-T-cell complex, and the cell suspension was then pipetted up and down several times and filtered. Total lung cells after RBC lysis with ACK lysis buffer
(150 $\mathrm{mM} \mathrm{NH}_{4} \mathrm{Cl}, 10 \mathrm{mM} \mathrm{KHCO}_{3}$ and $0.1 \mathrm{~mm}$ EDTA) were incubated with CD11c microbeads (Miltenyi Biotec, Auburn, Calif., USA) for $15 \mathrm{~min}$ at $4^{\circ} \mathrm{C}$. The cells were then washed, resuspended in cell separation buffer (Dulbecco's PBS) without $\mathrm{Ca}^{2+}$ and $\mathrm{Mg}^{2+}$ (Sigma) containing $0.5 \% \mathrm{BSA}$ and $2 \mathrm{~mm}$ EDTA, and passed through magnetic columns for the positive selection of CD11c+ lung cells. For LDC isolation, CD11c+ lung cells were subjected to sorting on a BD FACSAria II Cell Sorter (BD Biosciences, San Diego, Calif., USA). CD11 $\mathrm{c}^{\text {hi }}$ non-autofluorescent lung cells were gated as LDCs and sorted. CD11 $c^{\text {low }}$ autofluorescent lung cells that represent alveolar macrophages (AMs) were excluded in the sorting process. The purity of the sorted LDC was $>97 \%$ based on flow cytometric analysis. LDCs were stained with trypan blue and then counted using a hemocytometer.

\section{Flow Cytometry}

To analyze the expression of the various surface markers, the freshly prepared lung single-cell suspensions were subjected to CD11c-microbead selection. CD11c+lung cells were stained using anti-CD11c-APC, anti-MHC-II-PE (I-A/I-E), anti-CD40-PE, anti-CD80-PE, anti-F4/80-PE-Cy7 and anti-CD86-PE or with respective isotype controls (eBioscience, San Diego, Calif., USA). Fluorescence minus one controls were also used. For intracellular cytokine staining in the T cells, the lung cells were incubated at $5 \times$ $10^{6}$ cells/ml of complete RPMI medium containing $10 \%$ heat-inactivated FBS, $25 \mu \mathrm{g} / \mathrm{ml}$ gentamicin A, $2 \mathrm{mM}$ L-glutamine and $5 \times$ $10^{-5}$ M 2-ME (Kodak, Rochester, N.Y., USA) in 48-well plates at $37^{\circ} \mathrm{C}$, and stimulated with $50 \mathrm{ng} / \mathrm{ml}$ phorbol 12 -myristate 13 -acetate (Sigma) and $1 \mu \mathrm{g} / \mathrm{ml}$ ionomycin (Sigma). After $3 \mathrm{~h}$ of incubation, $20 \mu \mathrm{g} / \mathrm{ml}$ brefeldin A (Sigma) was added to accumulate the cytokines intracellularly, and samples were incubated for another $3 \mathrm{~h}$. Cultured cells were washed and incubated with FcR blocking antibodies (anti-16/32; eBioscience) for $15 \mathrm{~min}$ at $4{ }^{\circ} \mathrm{C}$ to block nonspecific staining. The cell surface markers were first stained with anti-CD3-FITC, anti-CD4-PE and anti-CD8-PE-Cy7 or respective isotype control antibodies (eBioscience). The cells were then fixed and permeabilized with Cytofix/Cytoperm (BD Pharmingen) according to the manufacturer's instructions, followed by intracellular staining with anti-IFN- $\gamma$-APC or isotype control antibodies (eBioscience). Finally, the cells were washed, resuspended in Dulbecco's PBS containing 2\% FCS and $1 \mathrm{~mm}$ EDTA and analyzed by flow cytomtery. Sample data were collected using a BD LSR II flow cytometer (BD Biosciences) and analyzed using FCS Express software (De Novo Software, Los Angeles, Calif., USA).

\section{Adoptive Transfer of LDCs and Challenge Infection}

For adoptive transfer, LDCs isolated from the lungs of uninfected/Cpn-infected KO or WT mice were first washed in proteinfree $\mathrm{PBS}$ and then inoculated intranasally into naïve recipient mice $\left(2 \times 10^{5} \mathrm{LDC} /\right.$ mouse $)$. Two hours after the adoptive transfer, mice were intranasally inoculated with $3 \times 10^{6}$ IFUs of Cpn in $40 \mu \mathrm{l}$ of PBS. The body weight of the mice before (day 0 ) and after inoculation were recorded daily. The mice were sacrificed on day 7 p.i. and the lung tissues were aseptically isolated and processed for the quantitative evaluation of Cpn in vivo growth as described previously [15]. For testing the cytokine levels in the lung tissues, the lungs were homogenized in $2 \mathrm{ml}$ of cold PBS. The supernatants were collected after centrifugation, and analyzed for the various cytokines by means of ELISA. 


\section{Histopathologic Analysis}

For histopathologic analysis, the lung tissues were fixed in $10 \%$ buffered formalin and embedded in paraffin. The tissue sections were stained with hematoxylin and eosin, and the histopathologic changes and cellular infiltration were assessed by light microscopy.

\section{Coculture of LDCs and T Cells}

To evaluate the ability of LDCs to activate Cpn-specific T-cell responses, the LDCs were cocultured with $\mathrm{T}$ cells isolated from the spleen of Cpn-immunized mice. For immunization, C57BL/6 mice were intranasally infected with Cpn $\left(3 \times 10^{6} \mathrm{IFUs}\right)$, and boosted 2 weeks later with the same dose. One week after the second immunization, $\mathrm{T}$ cells were isolated with high purity from the spleens of the immunized mice using negative selection by depleting the non-T cells, as described previously [15]. LDCs isolated from Cpn-infected KO or WT mice were cocultured with the purified T cells (LDC/T cell ratio $=1: 5)$ in $200 \mu \mathrm{l}$ of complete RPMI medium, with UK-EB $(1 \times$ $10^{4} \mathrm{IFUs} / \mathrm{ml}$ ) in 96 -well plates for $72 \mathrm{~h}$, and the concentrations of the various cytokines in the supernatants were measured by ELISA.

\section{Statistical Analysis}

Statistical analysis of data was performed using an unpaired two-tailed Student's t test (GraphPad Prism software v4, GraphPad, San Diego, Calif., USA). A p value $<0.05$ was considered significant.

\section{Results}

iNKT Cell Deficiency Alters the Number, Surface

Molecule Expression and Cytokine Production Pattern of LDCs following Cpn Infection

In order to assess the modulating effect of iNKT cells on LDCs, we first analyzed the number and surface molecule expression of the LDCs from KO and WT mice after Cpn infection. To characterize LDCs by flow cytometry, we used a combination of CD11c-expression and autofluorescence [23]. The flow cytometric FL-1/FITC detection channel was kept empty, i.e. there were no fluorochromeconjugated antibodies in the sample for this channel, to measure the autofluorescence. CD11c ${ }^{\text {hi }}$ non-autofluorescent LDCs were defined as LDCs and distinguished from the highly autofluorescent AMs based on their autofluorescence emission. We also used an antibody against the pan-macrophage marker F4/80 to confirm if CD11 $c^{\text {hi }}$ nonautofluorescent lung cells are indeed LDCs. Most CD11 $c^{\text {hi }}$ non-autofluorescent lung cells were $\mathrm{F} 4 / 80^{-}$(fig. 1a). We found that the frequency and absolute number of LDCs in the KO mice were lower than in the WT mice after infection; however, these numbers in the uninfected WT and $\mathrm{KO}$ mice were similar (fig. 1b, c). Although there was no difference in the frequency of AMs between uninfected WT and KO mice, it increased in the WT mice (compared to in the $\mathrm{KO}$ mice) at day 3 p.i. (fig. 1d). We also found a reduced accumulation of DCs in the MLNs of KO mice compared with WT mice p.i. (fig. 1e, f). We further analyzed the surface molecule expression on the LDCs. The LDCs from KO mice showed a reduced expression of MHC-II, CD80 and CD40 molecules compared to WT mice (fig. 2; table 1). On the other hand, the LDCs from KO mice showed a higher expression of CD86 compared to WT mice. Of note, the levels of surface marker expression on LDCs revealed no significant difference between uninfected WT and KO mice (data not shown).

The cytokine production pattern of DCs plays a critical role in skewing $\mathrm{T}$-cell responses. In particular, IL-12 production by DCs has been found to promote type-1 T-cell response important for protection against chlamydial infection. Therefore, to further test the functional implication of iNKT cells on the expansion and surface molecule expression of LDCs, we isolated WT-LDCs and KOLDCs with $>97 \%$ purity, excluding the AMs (fig. 3a). Upon culture, we found that KO-LDCs produced significantly lower levels of IL-12 (IL-12p40 and IL-12p70) than WT-LDCs. Conversely, IL-10 levels were significantly higher in the supernatants of cultured KO-LDCs, compared with those of WT-LDCs (fig. 3b). Taken together, these data suggest that iNKT cells can significantly modulate both the phenotype as well as the cytokine production pattern of LDCs after Cpn infection, particularly promoting IL-12 but regulating IL-10 production.

\section{iNKT Cells Modulate the Functional Ability of LDCs to Direct T-Cell Responses}

To directly examine the modulating effect of iNKT cells on LDC function in directing Cpn-specific T-cell responses, we cultured T cells from Cpn-immunized WT mice with KO-LDCs or WT-LDCs in the presence of UKEB stimulation. T cells cultured alone with UK-EB were used as controls. Upon analysis of culture supernatants by ELISA, we found that IFN- $\gamma$ production by T cells was significantly lower and IL-4 production significantly higher in KO-LDCs than in WT-LDCs (fig. 4a). Furthermore, we performed intracellular cytokine staining to specifically analyze the cytokine profile of CD4 and CD8 $\mathrm{T}$ cells following coculture with WT-LDCs or KO-LDCs. Flow cytometric analysis showed that WT-LDCs induced CD8 and CD4 T cells to produce large quantities of IFN $-\gamma$, which was significantly higher than that induced by KOLDCs (fig. 4b). Overall, these in vitro results demonstrate that iNKT cells modulate the functional ability of LDCs in polarizing $\mathrm{CD} 4$ and $\mathrm{CD} 8 \mathrm{~T}$ cells towards a type- 1 response that is necessary for immune protection against Cpn infection. 


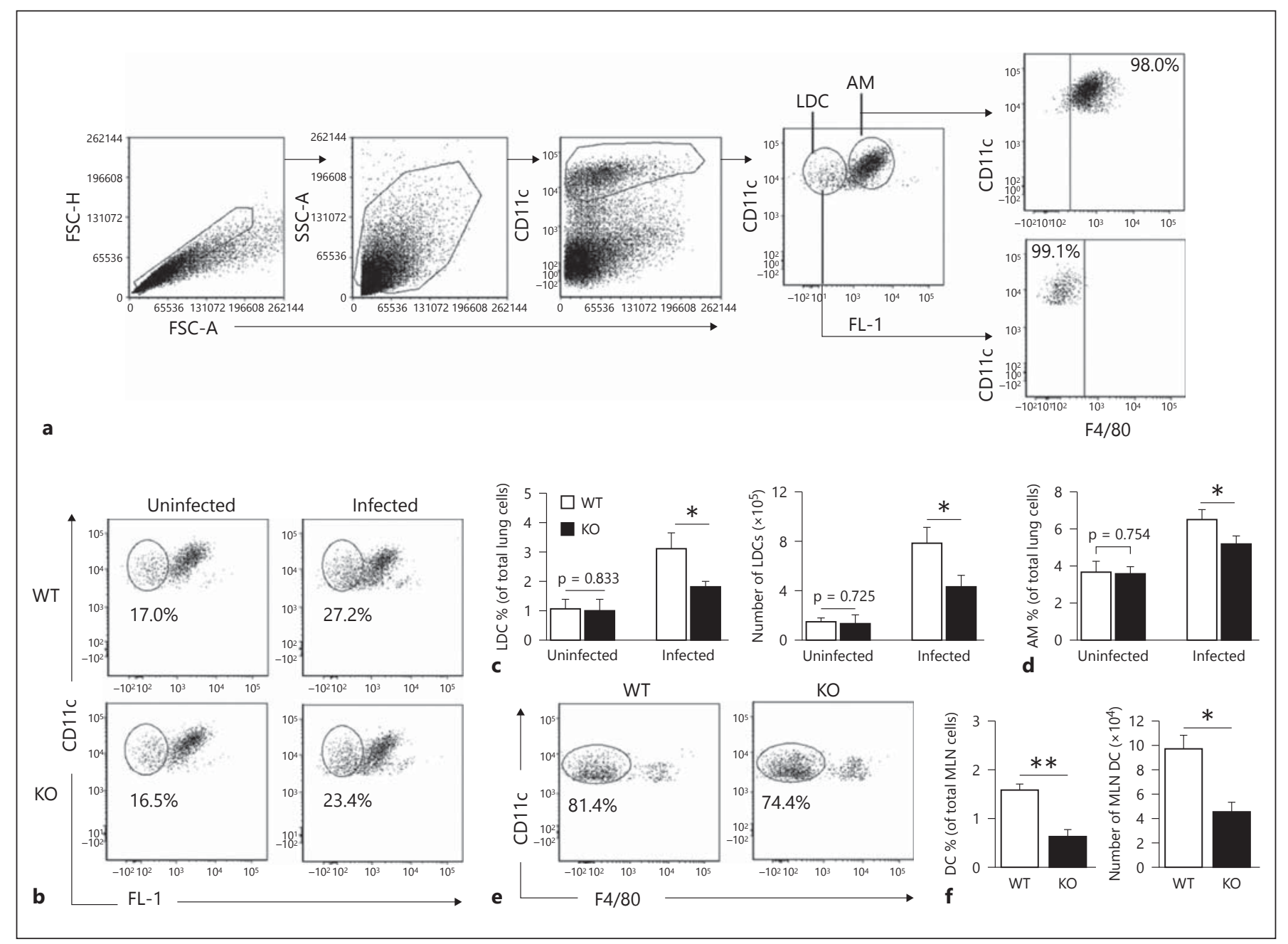

Fig. 1. LDCs in KO mice show reduced expansion after Cpn infection. KO and WT mice ( 3 in each group) were intranasally infected with Cpn $\left(3 \times 10^{6} \mathrm{IFUs} / \mathrm{mouse}\right)$ and sacrificed at day 3 p.i. Lungs and MLNs were harvested from the mice and processed into single-cell suspensions. a Flow cytometric gating strategy for analysis of LDCs. CD $11 c^{\text {hi }}$ non-autofluorescent and CD $11 c^{\text {low }}$ autofluorescent lung cells were gated and analyzed as LDCs and AMs, respectively. Analysis was performed on gated CD $11 c^{\text {hi }}$ non-autofluorescent lung cells. F4/80 was used to confirm whether CD11chi non-autofluorescent cells represent LDCs. b Representative flow cytometric images showing the frequency of LDCs

\section{Adoptive Transfer of LDCs from KO Mice Increases the Severity of Cpn Infection}

We further used the adoptive transfer approach to directly compare the functional capacity of WT-LDCs and KO-LDCs to induce protective immunity against Cpn challenge infection. We intranasally transferred WTLDCs or KO-LDCs to naïve recipient mice and then challenged them with Cpn infection. Mice receiving PBS after infection. c Graphs representing the percentage and absolute numbers of LDCs. The absolute numbers were calculated as the percentage of CD11 $c^{\text {hi }}$ non-autofluorescent lung cells $\times$ total number of lung cells/100. d Graphs representing the percentage of AMs of all the lung cells. e CD11c+F4/80- cells in the MLNs were analyzed as DCs. Flow cytometric images are shown. f Graphs representing the percentage and absolute numbers of DCs in the MLNs. Data are shown as mean \pm SD. One of the 2 independent experiments with similar results is shown. ${ }^{*} \mathrm{p}<0.05$; $* * \mathrm{p}<0.01$.

alone with the same challenge infection were used as controls. We found that the mice receiving KO-LDCs exhibited the most significant body weight loss compared with the WT-LDC recipients or even the PBS controls. On the other hand, WT-LDC-recipient mice lost less body weight and showed signs of earlier recovery (fig. 5Aa). The mice were sacrificed at day 7 p.i. and their lungs were harvested for the analysis of in vivo chlamydial growth 
Table 1. Summary of surface marker expression on LDCs from $\mathrm{WT}$ and $\mathrm{KO}$ mice following Cpn infection

\begin{tabular}{lrc}
\hline Surface markers & \multicolumn{1}{l}{ WT } & \multicolumn{1}{l}{ KO } \\
\hline CD40 & & \\
$\%$ & $57.2 \pm 2.9$ & $39.1 \pm 3.1^{*}$ \\
MFI & $178.7 \pm 2.5$ & $115.9 \pm 2.0^{* * *}$ \\
CD80 & & \\
$\%$ & $29.6 \pm 2.3$ & $21.4 \pm 1.6^{*}$ \\
MFI & $36.8 \pm 2.1$ & $27.9 \pm 2.3$ \\
CD86 & & \\
$\%$ & $14.9 \pm 1.0$ & $22.5 \pm 1.0^{* *}$ \\
MFI & $15.3 \pm 1.3$ & $23.9 \pm 2.4^{*}$ \\
MHC-II & & \\
$\%$ & $91.0 \pm 1.1$ & $86.9 \pm 1.0^{*}$ \\
MFI & $571.8 \pm 13.5$ & $500.7 \pm 8.8^{*}$ \\
\hline
\end{tabular}

Three mice from each group were sacrificed at day 3 after Cpn infection. CD11c+ lung cells were isolated by CD11c-magnetic microbead selection. CD $11 \mathrm{c}^{\text {hi }}$ non-autofluorescent lung cells were gated as LDCs as described in figure 1a. The surface molecule expression on LDCs was analyzed by flow cytometry. Statistical analyses were performed using a Student's t test. Data are shown as mean $\pm \mathrm{SD}$. One of the 2 independent experiments with similar results is shown. MFI $=$ Mean fluorescence intensity. ${ }^{*} \mathrm{p}<0.05$; ${ }^{* *} \mathrm{p}<0.01{ }^{* * *} \mathrm{p}<0.001$.

and lung histopathology. We found that the Cpn loads in the lungs of KO-LDC recipients were significantly higher than in the WT-LDC recipients and controls (fig. 5Ab). Of note, no viable chlamydial organisms were found in the WT-LDCs and KO-LDCs, and therefore the increased infection in $\mathrm{KO}-\mathrm{LDC}$ recipients was unlikely due to the potential trace amount of Cpn organisms carried by the transferred LDCs. Furthermore, we found that the most severe inflammatory and pathologic changes of all the groups occurred in the lungs of the KO-LDC recipients (fig. 5Ac). To rule out the possibility of whether LDCs from uninfected mice have any impact on the outcome of T-cell immunity, we pooled the LDCs from uninfected WT or KO mice and transferred them to naïve recipient mice. Upon challenge infection, the mice receiving LDCs from WT or KO mice and PBS recipients showed no significant difference in their loss of body weight, chlamydial burden and lung pathology (fig. 5Ba, $\mathrm{Bb}, \mathrm{Bc}$ ). These in vivo data indicate that iNKT cells are critically important for enhancing LDC function to induce protective immunity against Cpn infection. Without iNKT cell modulation, LDCs not only fail to promote protective immunity but also increase the infection and the pathology.

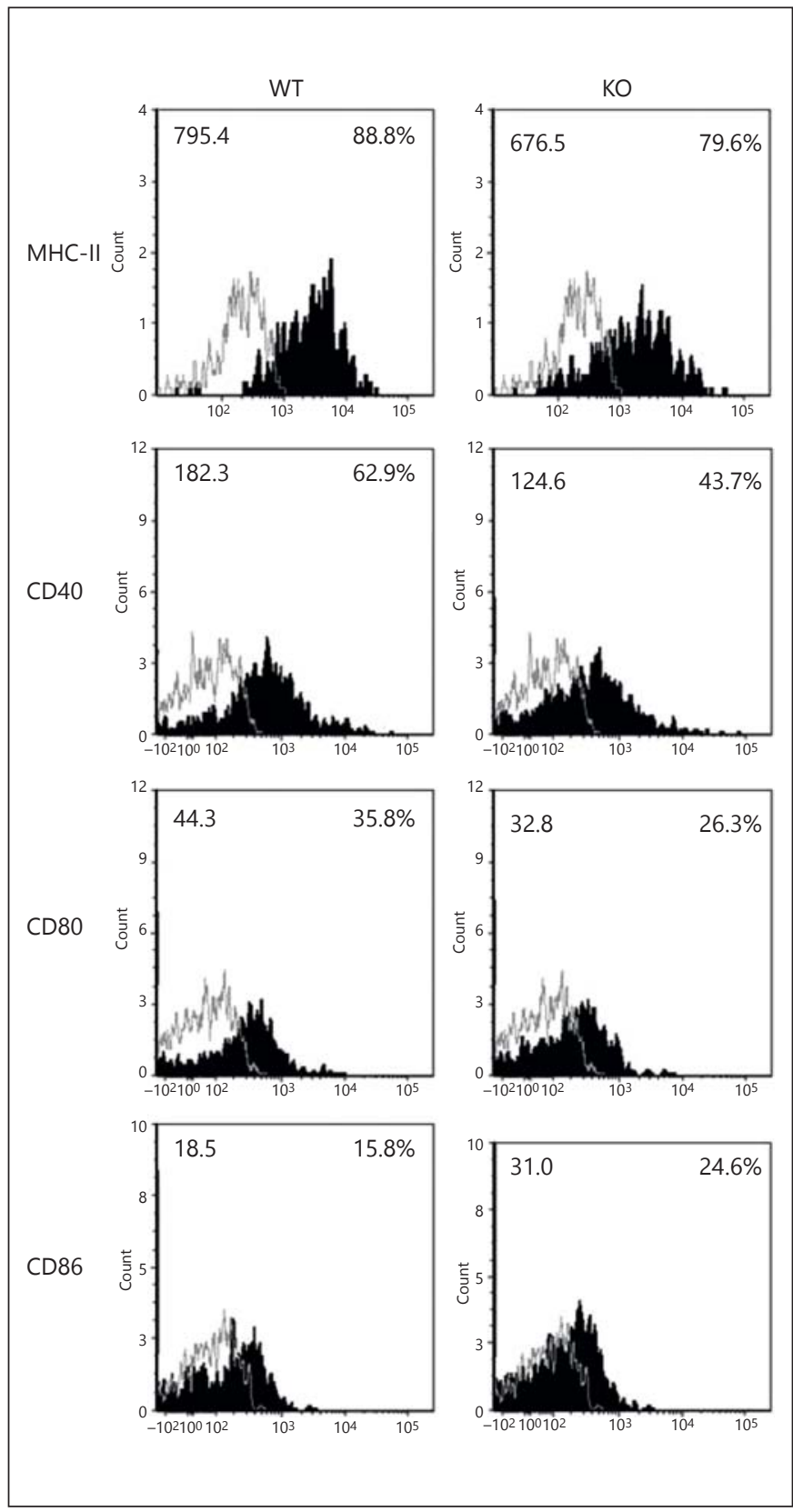

Fig. 2. iNKT cells alter LDC surface molecule expression after infection. Both $\mathrm{KO}$ as well as WT mice ( 3 in each group) were sacrificed at day 3 after intranasal infection $\left(3 \times 10^{6} \mathrm{IFUs} /\right.$ mouse $)$. Lungs were harvested and processed into single-cell suspensions. CD11c+ lung cells were isolated by using CD11c-magnetic microbead selection. CD11 $c^{\text {hi }}$ non-autofluorescent lung cells were gated as LDCs as shown in figure 1a. The LDCs were analyzed for the expression of MHC-II and costimulatory molecules by flow cytometry. Flow cytometric dotplots are shown. Expressions of CD80, CD86, CD40 and MHC-II on LDCs (shaded histogram) and isotype control (line) were shown. The mean fluorescence intensity (left) and percentages of positive cells (right) were indicated. One of the 2 independent experiments with similar results is shown. 
Fig. 3. Analysis of purity and cytokine profile of sorted LDCs following infection. Lungs were harvested from mice at day 3 p.i., processed into single-cell suspensions and enriched with CD11c magnetic beads using an MACS column. The CD11c-enriched lung cells were stained with APCconjugated-anti-CD11c or isotype control antibodies. Flow cytometric analysis was performed on gated CD $11 \mathrm{c}+$ cells. CD $11 \mathrm{c}^{\text {hi }}$ non-autofluorescent cells were considered as LDCs and sorted by flow cytometry as described in materials and methods. a Flow cytometric dotplots showing the gating strategy and the purity of sorted LDCs using antibodies for CD11c and F4/80. b LDCs purified from $\mathrm{KO}$ or WT mice were placed in culture for $72 \mathrm{~h}$, and the concentrations of IL-12p40, IL-12p70 and IL-10 in the supernatants were measured by ELISA. Data are shown as mean \pm SD. One of the 2 independent experiments with similar results is shown. ${ }^{* *} \mathrm{p}<0.01$; $^{* * *} \mathrm{p}<0.001$.

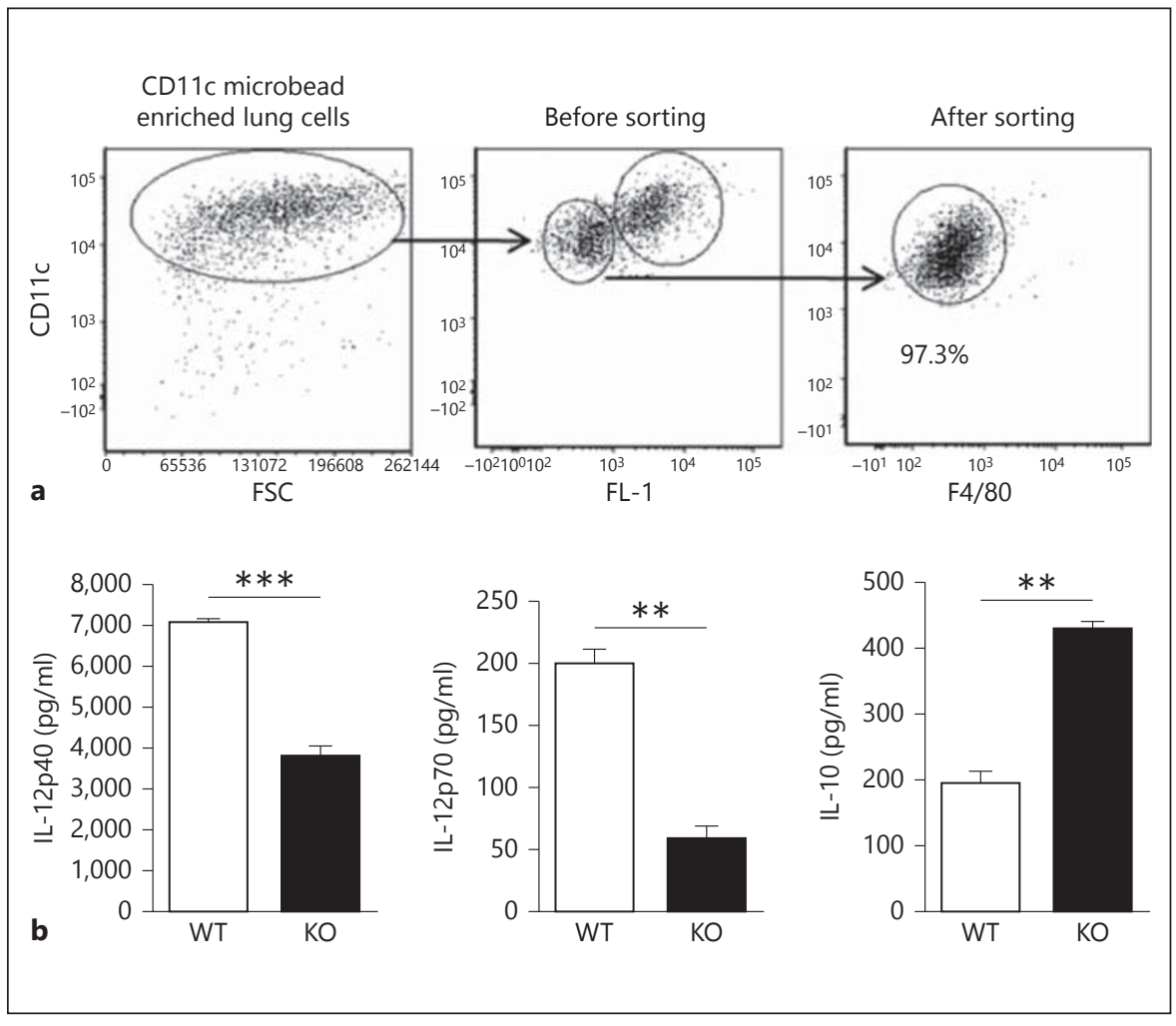

Adoptive Transfer of LDCs from $\mathrm{KO}$ and

WT Mice Generates Differential Cytokine

Production Patterns in Recipient Mice after

Challenge Infection

To examine the immunological basis for the distinct effect of adoptively transferred KO-LDCs and WT-LDCs, we analyzed the cytokine production pattern in the lung tissues and MLNs of the LDC-recipient mice at day 7 following Cpn challenge. We found that the mice receiving KO-LDCs showed an increased type- 2 response with significantly higher levels of IL-4 and IL-10, whereas those receiving WT-LDCs showed an increased type-1 response with enhanced levels of IFN- $\gamma$, IL-12p40 and IL12 p70 (fig. 6, 7). In addition, the KO-LDC-recipient mice had lower levels of TNF- $\alpha$ in their lungs than in the WT$\mathrm{LDC}$ recipients or PBS control mice (fig. 7). To further investigate the specific T-cell cytokine profile, we performed intracellular cytokine analysis of the lung T cells by flow cytometry. A greater number of IFN $-\gamma+\mathrm{T}$ cells were found in the recipients of WT-LDC than in KOLDC recipients or controls (fig. 8). Together, these data suggest that WT-LDCs, in contrast to KO-LDCs, significantly induce a type-1 polarized immune response in vivo.

\section{Discussion}

In this study, we demonstrate a promoting effect of iNKT cells on LDC function in generating protective Tcell immunity to Cpn infection using various experimental approaches. Specifically, we found that iNKT cells induced the expansion of LDCs following Cpn lung infection and, more importantly, enhanced the expression of costimulatory molecules and cytokine production by LDCs. Furthermore, we found that WT-LDCs promoted protective type-1 T-cell responses whereas KO-LDCs augmented type-2 $\mathrm{T}$-cell response as assessed in in vitro LDC:T-cell coculture experiments. Moreover, upon adoptive transfer, WT-LDCs enhanced the clearance of lung infection, while KO-LDCs increased the severity of the infection. This was associated with a significant differential effect on the lung cytokine pattern in the corresponding recipient mice; KO-LDC-recipient mice exhibited increased type- 2 cytokine production while WTLDC recipients reflected a type- 1 cytokine profile with enhanced IFN- $\gamma$ production. Altogether, the in vitro and in vivo data suggest that iNKT cells play a critical role in conferring protective immunity against Cpn infection through modulating the function of LDCs. 
Fig. 4. iNKT cells modulate LDC function in polarizing $\mathrm{T}$ cells towards type- 1 responses. $\mathrm{T}$ cells from Cpn-immunized mice were isolated and cocultured with KO-LDCs or WT-LDCs in the presence of UK-EB stimulation. a The concentrations of type-1 (IFN- $\gamma$ ) and type-2 (IL-4) cytokines in the culture supernatants were measured by ELISA. b Intracellular cytokine production by $\mathrm{T}$ cells in LDC:T-cell coculture. Analysis was performed on gated CD3+ cells. Representative flow cytometric images (left) and graphs (right) representing the summary of the percentage of IFN- $\gamma+$ CD4, CD8 and CD3 T cells. Data are shown as mean \pm SD. Two independent experiments with 4 mice in each group were performed, and 1 representative experiment is shown. ${ }^{*} \mathrm{p}<0.05$; ${ }^{* *} \mathrm{p}<0.01$; *** $\mathrm{p}<0.001$.

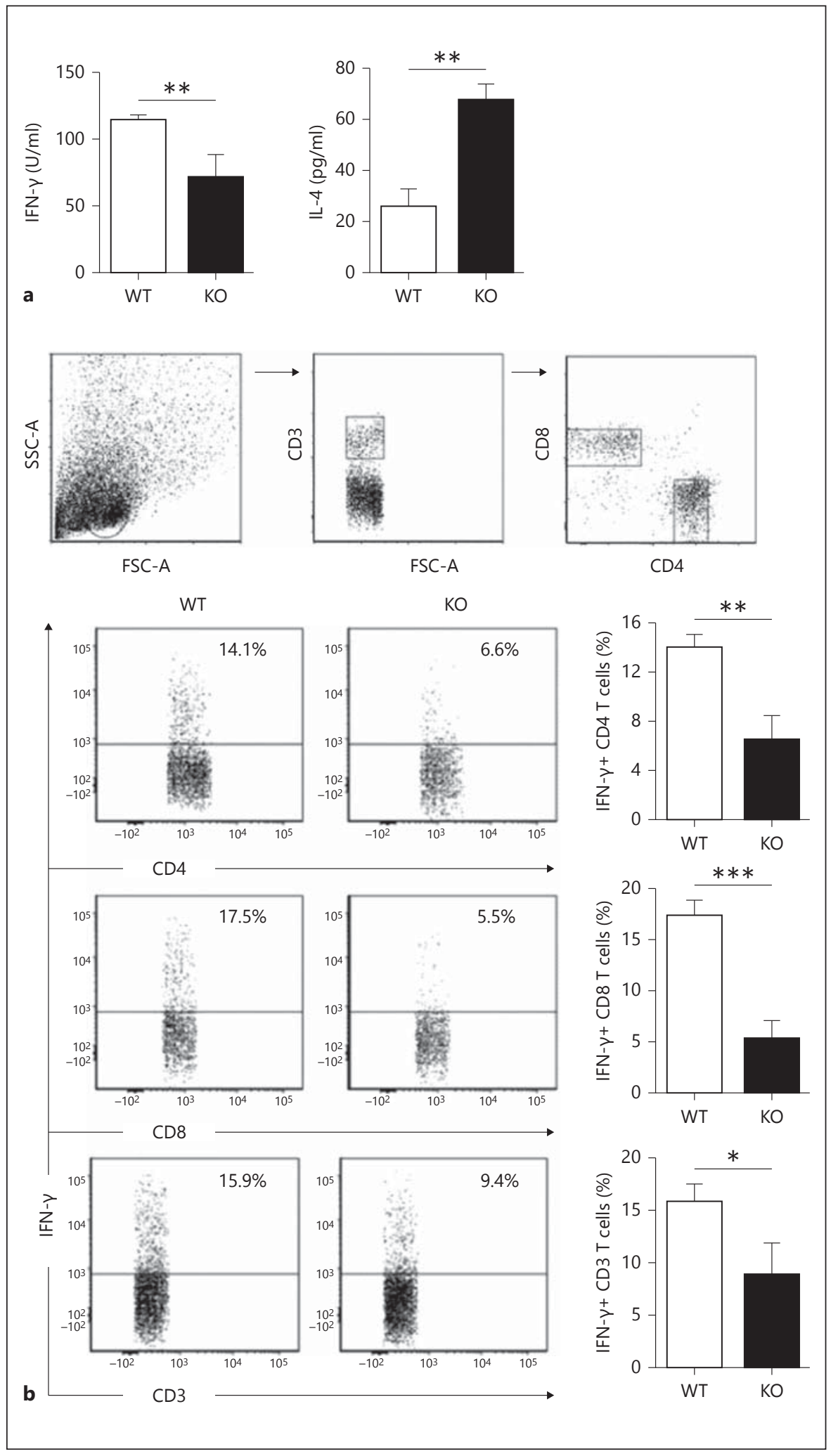

J Innate Immun 2015;7:260-274 DOI: $10.1159 / 000368779$ 
Fig. 5. Adoptive transfer of KO-LDCs and WT-LDCs to test their function in vivo. Naïve mice (C57BL/6) were intranasally delivered KO-LDCs or WT-LDCs and then challenged with Cpn infection $\left(3 \times 10^{6}\right.$ IFUs/mouse). Mice receiving PBS only with the same challenge infection were considered as controls. All mice were sacrificed at day 7 p.i. Lungs from the recipient mice were homogenized and tested for chlamydial loads. The mean of the $\log _{10^{-}}$ transformed IFUs/lung is shown. Lungs from recipient mice were examined for pathology by HE staining and analyzed under light microscope. A Transfer of KO-LDCs and WT-LDCs isolated from Cpn-infected mice. a Body weight loss. b In vivo chlamydial growth. c Lung pathology. B Transfer of KO-LDCs and WT-LDCs isolated from uninfected mice. a Loss of body weight. b Chlamydial burden. c Lung pathology. Data are shown as mean \pm SD. Two independent experiments with 3-4 mice in each group were performed, and 1 representative experiment is shown. ${ }^{*} \mathrm{p}<$ 0.05 ; $^{* *} \mathrm{p}<0.01$; $^{* * *} \mathrm{p}<0.001$.
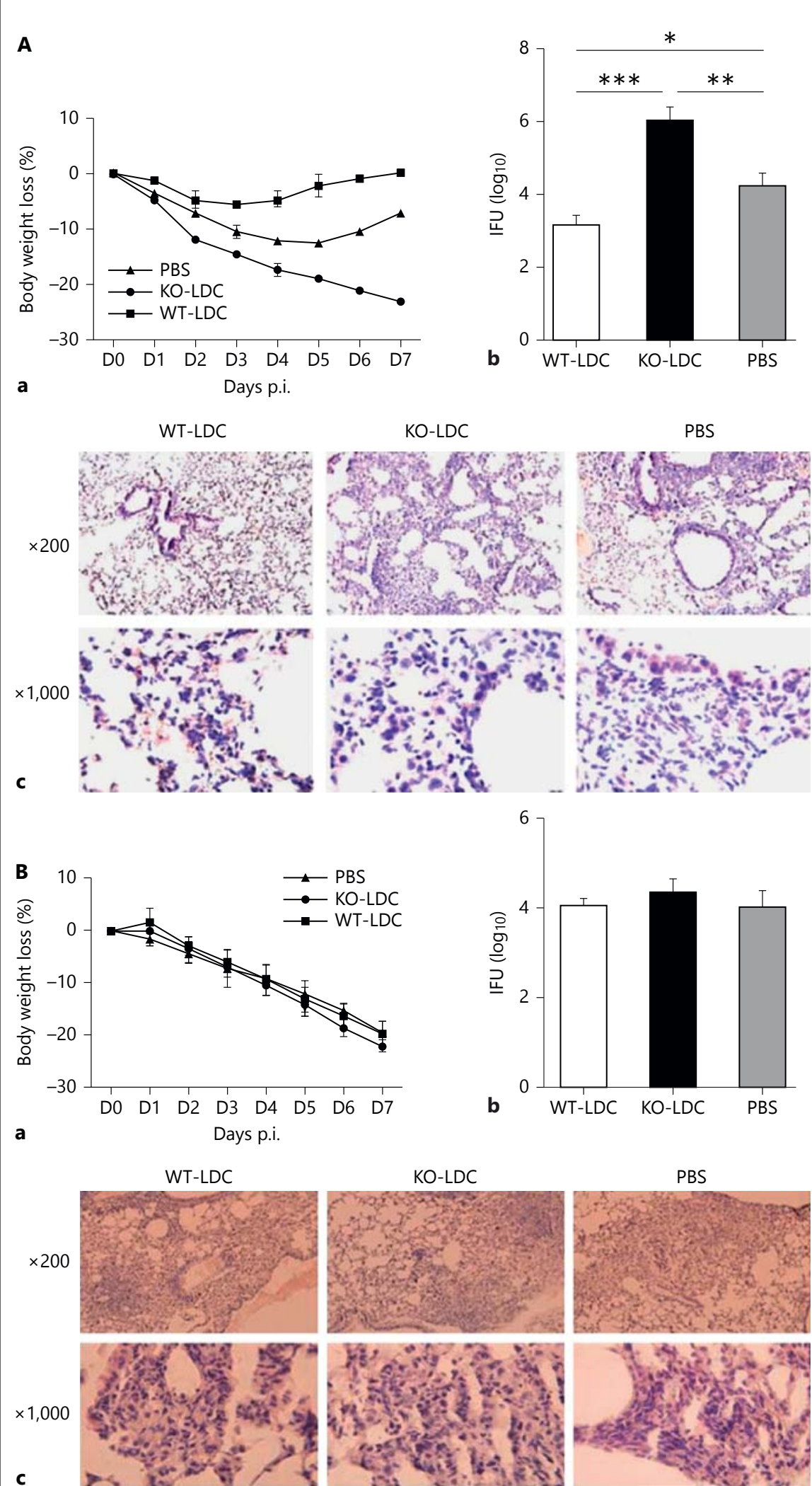

KO-LDC
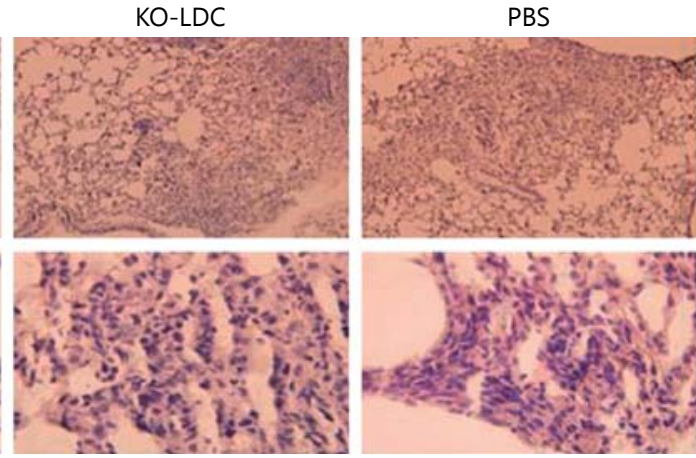

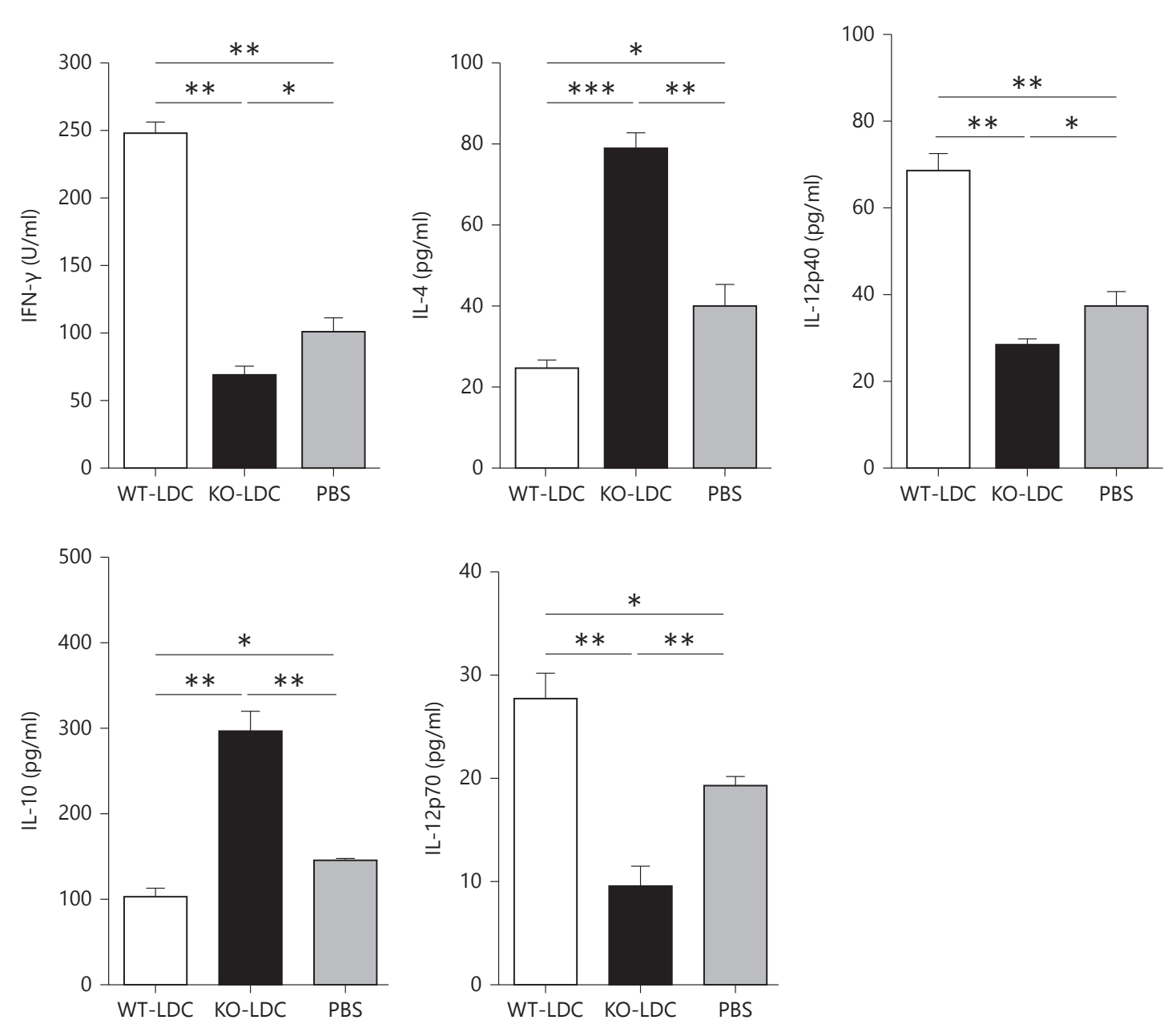

Fig. 6. Adoptive transfer of KO-LDCs induces a type-2 response in the MLNs of recipient mice. Recipient mice were sacrificed at day 7 p.i. MLNs were harvested from the mice and processed into singlecell suspensions. MLN cells were cultured with UK-EB for $72 \mathrm{~h}$. The cytokine levels in the culture supernatants were examined by ELISA. Data are shown as mean \pm SD. Two independent experiments with 4 mice in each group were performed, and 1 representative experiment is shown. ${ }^{*} \mathrm{p}<0.05$; $^{* *} \mathrm{p}<0.01$; $^{* *} \mathrm{p}<0.001$.
The key aspect derived from the results of this study is the modulating effect of iNKT cells on the function of major APCs residing at the local site during a respiratory tract infection with Cpn, a clinically relevant human pathogen known to cause a wide variety of respiratory disorders. Our previous findings demonstrated that the KO mice showed exacerbated susceptibility to primary Cpn infection [24]. Following Cpn infection, these mice had greater body weight loss, higher lung bacterial loads and more intense inflammatory and pathologic changes in their lungs compared to the WT controls. Moreover, the KO mice compared to WT mice showed dramatically reduced type- $1 \mathrm{~T}$ cell responses, showing that iNKT cells influence the development of protective adaptive immune responses. Overall, the findings suggested that the lack of iNKT cell activity in the KO mice significantly altered the pulmonary environment and immune responses following Cpn infection. These changes can alter the infiltration/differentiation/activation status of LDCs which play a key role in the development of protective immune responses. Indeed, the present findings showed that iNKT cell deficiency induced phenotypic and functional changes in LDCs following Cpn infection. Furthermore, we found that the intranasal adoptive transfer of WT-LDCs in naïve recipient mice conferred strong protective immunity, whereas 

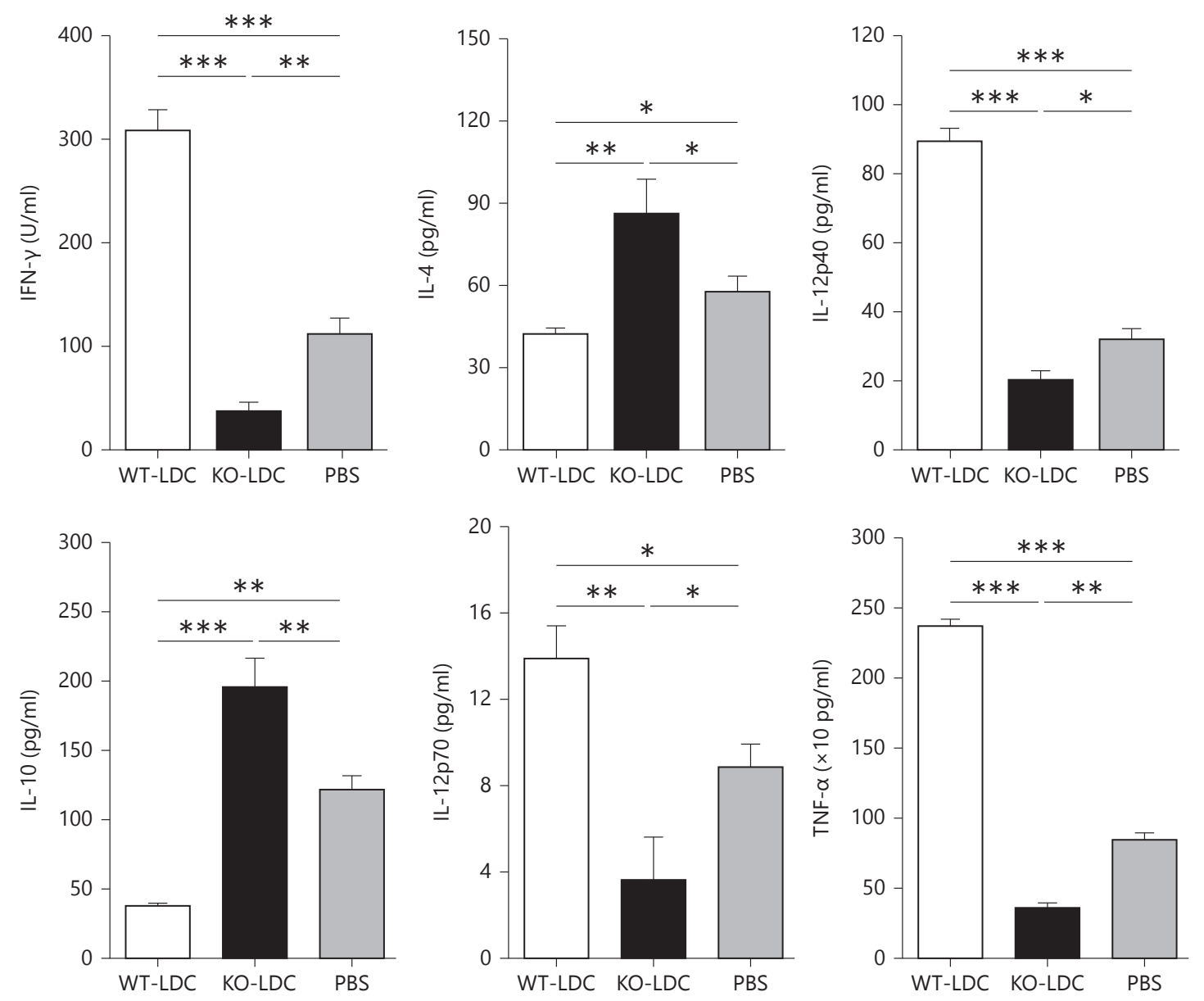

Fig. 7. Effect of LDC adoptive transfer on the local cytokine environment in the lungs of recipient mice. Mice receiving LDCs or PBS were sacrificed at day 7 p.i. Lungs were harvested from the mice, homogenized in cold, protein-free PBS and centrifuged. Lev- els of different cytokines in the supernatants were measured by ELISA. Data are shown as mean \pm SD. Two independent experiments with 4 mice in each group were performed, and 1 representative experiment is shown. ${ }^{*} \mathrm{p}<0.05$; $^{* *} \mathrm{p}<0.01$; $^{* * *} \mathrm{p}<0.001$. transfer of KO-LDCs promoted pathology after chlamydial challenge infection. However, the transfer of LDCs from uninfected $\mathrm{KO}$ and WT mice did not have any impact on the outcome of infection. Altogether, these findings highlight the significance of in vivo interactions between iNKT cells and LDCs for the development of protective immunity against an intracellular bacterial infection. In particular, the differential effects observed following transfer of LDCs from infected mice compared to uninfected mice demonstrate iNKT cell responses playing an important role in programming LDCs during the primary infection in determining the outcome of a secondary infection. These findings have important implications in vaccine development. In order to execute their protective role in host defense against chlamydial infection, LDCs need help from iNKT cells which can tune the function of LDC to polarize $\mathrm{T}$ cells towards a protective type- 1 response. In the absence of this help, the LDCs switch to a 'DC2' mode that leads to the generation of type- 2 responses and pathology. The functional implication of the iNKT cellLDC interaction is that it confers protective immunity as well as preventing pathologic changes. The mechanism of how iNKT cells impact the SDC function during Cpn infection has been investigated in our previous study, where we found that iNKT cells modulate DC re- 
Fig. 8. Local T-cell cytokine production in the lungs of recipient mice. Mice receiving KO-LDCs, WT-LDCs or PBS were sacrificed at day 7 p.i. Lung cells from the recipient mice were cultured at the concentration of $5 \times 10^{6}$ cells in complete RPMI medium. The cytokine production pattern in the T cells was analyzed by intracellular cytokine staining. Analysis was performed on gated CD3+ cells as described in figure 4b. a Representative flow cytometric images showing the percentages of IFN- $\gamma+$ CD4 and CD 3 T cells. b Graphical summary of the percentages and absolute numbers of IFN- $\gamma+$ T cells. The absolute numbers of IFN- $\gamma+$ T cells were calculated as percentage of IFN $-\gamma+$ T cells $\times$ total number of cells per lung/100. Data are shown as mean \pm SD. Two independent experiments with 4 mice in each group were performed, and 1 representative experiment is shown. ${ }^{*} \mathrm{p}<$ $0.05 ;{ }^{* *} \mathrm{p}<0.01$; *** $\mathrm{p}<0.001$.

(For figure $8 b$ see next page.)

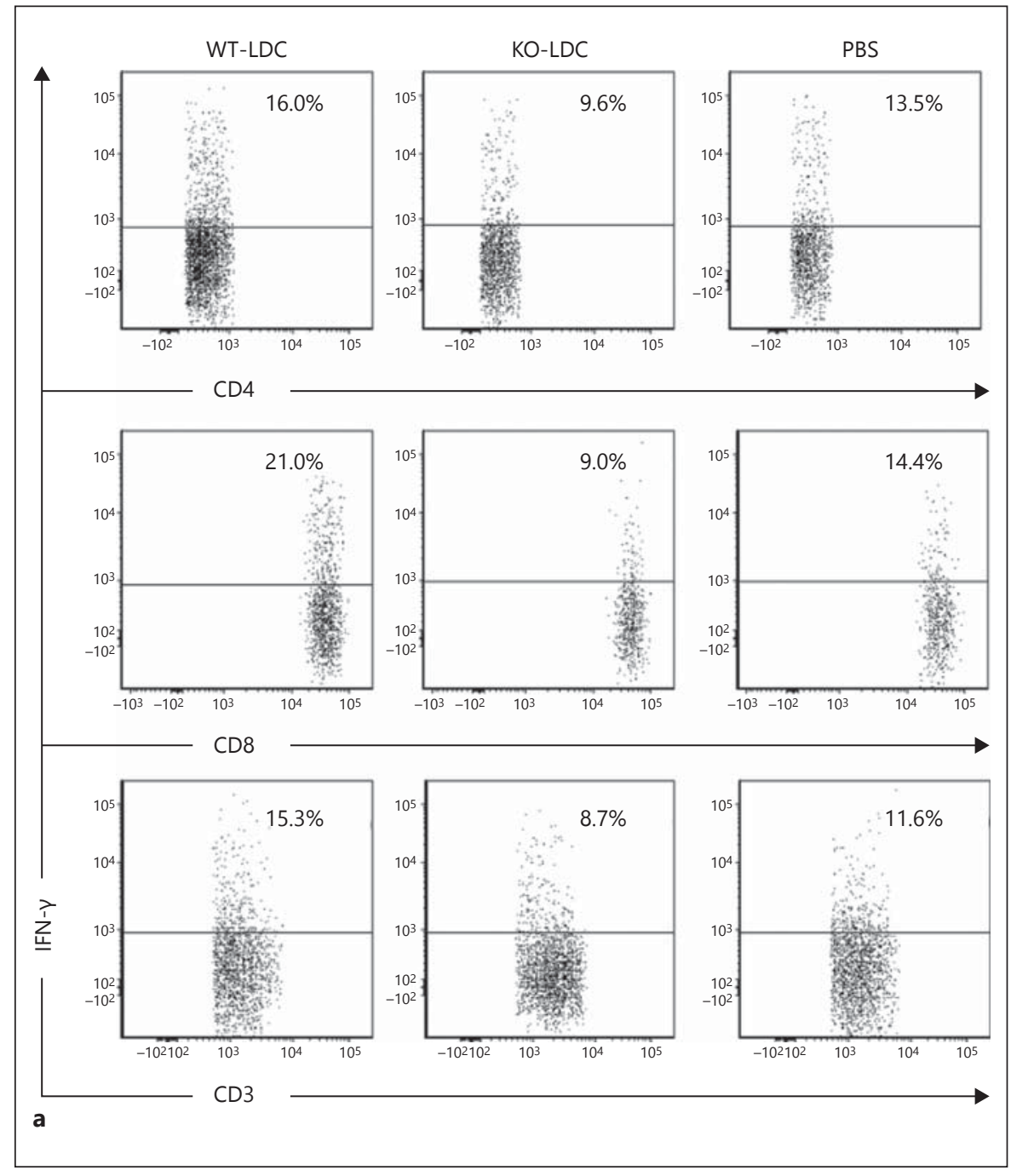

sponses in a CD40L-, IFN- $\gamma$ - and cell-cell contact-dependent fashion [15]. It should be noted that this study has examined LDCs only at one time point following Cpn infection. It is still possible that the effect of iNKT cells on LDCs and SDCs could be quantitatively and qualitatively different at different stages of infection.

The effect of iNKT cells on LDCs is reflected by the cell number, surface markers and cytokine pattern. We found a markedly reduced expansion of DCs in the lung and the MLNs of KO mice following lung infection, as shown by the lower percentage and number of DCs in these tissues. However, it should be noted that the number of LDCs in KO mice also increased following chlamydial infection, suggesting that the expansion of LDCs was not dependent on the iNKT cells, although these cells could promote LDC function. Furthermore, KO-
LDCs exhibited lower expression of MHC-II, CD40 and CD80 molecules compared with that of WT-LDCs. These findings are largely consistent with those of a recent study by Paget et al. [22], where a major population of LDCs, i.e. CD103+ LDCs, was significantly reduced in $\mathrm{KO}$ mice, and showed reduced expression of the costimulatory molecule, CD40, after influenza A virus infection. They also demonstrated reduced expression of CD86 following chlamydial infection as opposed to the increased expression in our study. This indicates that different pathogens may modulate the expression of some costimulatory molecules in diverse ways. Although the role of costimulatory molecules on DCs in promoting a type- 1 response is widely appreciated, some studies suggest that their function may overlap in modulating Th1/Th2 differentiation, particularly in the 

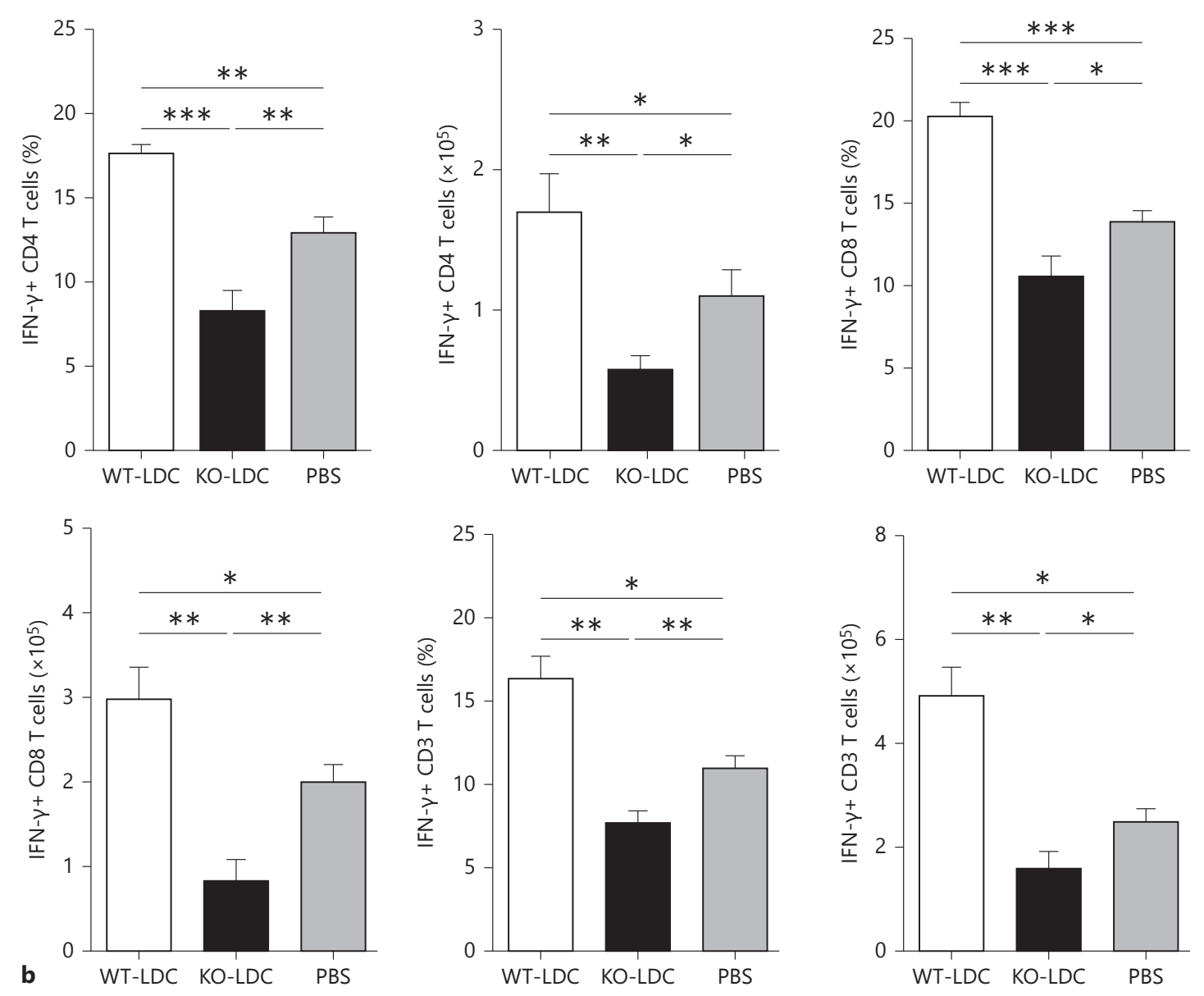

8

case of CD86 [25, 26]. Moreover, it has been reported that CD86 expression is important for type- 2 responses; this matches the data from our study which showed an elevated type- 2 response in the KO-LDC-recipient mice. It is also possible that the impact of elevated CD86 expression on LDCs in $\mathrm{KO}$ mice is a reflection of the reduced expression of CD80, CD40 and MHC-II molecules on the final outcome of T-cell responses. The cytokine production pattern of DCs is more relevant to their function of directing T-cell responses. Our data on the cytokine pattern of LDCs further support the importance of iNKT cells on LDC function. KO-LDCs produced significantly lower levels of IL-12 (IL-12p70 and IL-12p40), which is a type-1 promoting cytokine. In contrast, the IL-10 production by LDCs was significantly higher in the KO mice than in the WT mice. The detrimental role of IL-10 in the host defense against chlamydial infections has been demonstrated by many stud- ies $[27,28]$. Thus, our overall findings demonstrate that iNKT cells activated by local (lung) infection can comprehensively influence LDCs in their expansion, phenotype and cytokine profile.

Importantly, our data provided direct in vivo evidence on the effect of iNKT cells on LDC function. Given the critical role of cytokines in generating protective responses against chlamydial infections, we examined the effect of iNKT cells on the modulation of LDC function in influencing the local cytokine environment and thus the outcome of the infection. Intranasal adoptive transfer of WT-LDCs and KO-LDCs showed a differential effect on the cytokine production pattern in the local tissues, particularly the lungs and MLNs of the recipient mice after the challenge infection. The WT-LDC recipients had higher levels than the KO-LDC recipients of the cytokines that induce type- 1 immunity. Of note, our results showed an enhanced production of TNF- $\alpha$ in the lungs of mice 
receiving WT-LDCs compared to the mice that received KO-LDCs or PBS; this is in line with the finding that TNF- $\alpha$ inhibits Cpn growth [29]. Furthermore, we analyzed both CD4 and CD8 T-cell responses. Previous studies suggest that CD8 $\mathrm{T}$-cell responses are crucial for controlling Cpn infection, although its role in C. trachomatis is still debatable [30, 31]. Accordingly, KO-LDC-recipient mice exhibited significantly lower levels of lung IFN- $\gamma+$ CD8 T cells than recipients of WT-LDCs or the PBS controls. In correlation with the in vivo findings, our in vitro data also showed reduced IFN- $\gamma$ production by CD4 and CD8 T cells upon coculture with KO-LDCs compared to with WT-LDCs.

In summary, our study illustrates the critical role of iNKT cells in conferring protective immunity against Cpn infection by modulating the function of LDCs which are specialized to act primarily at the local site of infection and initiate effective mucosal immunity against respira- tory pathogens. Our findings may well have implications for exploring the immune mechanisms that enhance host immunity to chlamydial lung infections and the control of the long-term complications of these infections, such as atherosclerosis and Alzheimer's disease.

\section{Acknowledgements}

This work was supported by grants from the Canadian Institutes of Health Research (CIHR), Manitoba Health Research Council (MHRC) and Manitoba Institute of Child Health (MICH) to X.Y., who was the Canada Research Chair in Infection and Immunity. S.S. is the recipient of an MHRC/MICH graduate studentship.

\section{Disclosure Statement}

The authors declare that there are no competing financial interests.

\section{References}

$\checkmark 1$ Condon TV, Sawyer RT, Fenton MJ, Riches DWH: Lung dendritic cells at the innateadaptive immune interface. J Leuk Biol 2011; 90:883-895.

-2 Vermaelen K, Pauwels R: Pulmonary dendritic cells. Am J Respir Crit Care Med 2005;172: 530-551.

$>3$ Dodge L, Carr MW, Cernadas M, Brenner MB: IL-6 production by pulmonary dendritic cells impedes Th1 immune responses. J Immunol 2003;170:4457-4464.

4 Stumbles PA, Thomas JA, Pimm CL, Lee PT, Venaille TJ, Proksch S, Holt PG: Resting respiratory tract dendritic cells preferentially stimulate $\mathrm{T}$ helper cell type 2 (Th2) responses and require obligatory cytokine signals for induction of Th1 immunity. J Exp Med 1988; 188:2019-2031.

5 Lagranderie M, Nahori MA, Balazuc AM, Kiefer-Biasizzo H, Lapa e Silva JR, Milon G, Marchal G, Vargaftig BB: Dendritic cells recruited to the lung shortly after intranasal delivery of $\mathrm{Myco}$ bacterium bovis BCG drive the primary immune response towards a type 1 cytokine production. Immunology 2003;108:352-364.

-6 McGill J, Van Rooijen N, Legge KL: Protective influenza-specific CD8 $\mathrm{T}$ cell responses require interactions with dendritic cells in the lungs. J Exp Med 2008;205:1635-1646.

$>7$ Von Garnier C, Nicod LP: Immunology taught by lung dendritic cells. Swiss Med Wkly 2009;139:186-192.

$>8$ Bendelac A, Savage PB, Teyton L: The biology of NKT cells. Annu Rev Immunol 2007;25: 297-336.

-9 Godfrey DI, Rossjohn J: New ways to turn on NKT cells. J Exp Med 2011;208:1121-1125.
10 Peng Y, Zhao L, Shekhar S, Liu L, Wang H, Chen Q, Gao X, Yang X, Zhao W: The glycolipid exoantigen derived from Chlamydia muridarum activates natural killer $\mathrm{T}$ cells. Cell Mol Immunol 2012;9:361-366.

11 Brigl M, Tatituri RV, Watts GF, Bhowruth V, Leadbetter EA, Barton N, Cohen NR, Hsu FF, Besra GS, Brenner MB: Innate and cytokinedriven signals, rather than microbial antigens, dominate in natural killer $\mathrm{T}$ cell activation during microbial infection. J Exp Med 2011; 208:1163-1177.

12 Belland R, David M, Byrne GI: Chlamydia. Nat Rev Immunol 2004;2:530-531.

-13 Kuo CC, Jackson LA, Campbell LA, Grayston JT: Chlamydia pneumoniae (TWAR). Clin Microbiol Rev 1995;8:451-461.

$\checkmark 14$ Burillo A, Bouza E: Chlamydophila pneumoniae. Infect Dis Clin N Am 2010;24:61-71.

15 Joyee AG, Qui H, Fan Y, Wang S, Yang X: Natural killer T cells are critical for dendritic cells to induce immunity in Chlamydia pneumoniae. Am J Respir Cri Care Med 2008;178: $745-756$

16 Joyee AG, Uzonna J, Yang X: Invariant NKT cells preferentially modulate the function of CD8a+ dendritic cell subset in inducing type 1 immunity against infection. J Immunol 2010;184:2095-2106.

-17 Masten BJ, Yates JL, Pollard Koga AM, Lipscomb MF: Characterization of accessory molecules in murine lung dendritic cell function: roles for CD80, CD86, CD54, and CD40. Am J Respir Cell Mol Biol 1997;16: 335-342.

18 Ballesteros-Tato A, León B, Lund FE, Randall TD: Temporal changes in dendritic cell sub- sets, cross-priming and costimulation via CD70 control CD8(+) T cell responses to influenza. Nat Immunol 2010;11:216-224.

19 Lukens MV, Kruijsen D, Coenjaerts FE, Kimpen JL, van Bleek GM: Respiratory syncytial virus-induced activation and migration of respiratory dendritic cells and subsequent antigen presentation in the lungdraining lymph node. J Virol 2009;83: 7235-7243.

20 Kim TS, Braciale TJ: Respiratory dendritic cell subsets differ in their capacity to support the induction of virus-specific cytotoxic CD8+ T cell responses. PLoS One 2009;4:e4204.

21 Ivanov S, Fontaine J, Paget C, Macho Fernandez E, Van Maele L, Renneson J, Maillet I, Wolf NM, Rial A, Leger H, Ryffel B, Frisch B, Chabalgoity JA, Sirard JC, Benecke A, Faveeuw C, Trottein E: Key role for respiratory CD103+ dendritic cells, IFN- $\gamma$, and IL-17 in protection against Streptococcus pneumoniae infection in response to $\alpha$-galactosylceramide. I Infect Dis 2012;206:723-734.

22 Paget C, Ivanov S, Fontaine J, Blanc F, Pichavant $\mathrm{M}$, Renneson J, Bialecki E, Pothlichet J, Vendeville C, Barba-Spaeth G, Huerre MR, Faveeuw C, Si-Tahar M, Trottein F: Potential role of invariant NKT cells in the control of pulmonary inflammation and CD8+ T cell response during acute influenza $\mathrm{A}$ virus H3N2 pneumonia. J Immunol 2011;186: 5590-5602.

23 Vermaelen K, Pauwels R: Accurate and simple discrimination of mouse pulmonary dendritic cell and macrophage populations by flow cytometry: methodology and new insights. Cytometry A 2004;61:170-177. 
24 Joyee AG, Qiu H, Wang S, Fan Y, Bilenki L, Yang X: Distinct NKT cell subsets are induced by different Chlamydia species leading to differential adaptive immunity and host resistance to the infections. J Immunol 2007;178:1048-1058.

25 Bagenstose LM, Class R, Salgame P, Monestier M: B7-1 and B7-2 co-stimulatory molecules are required for mercury-induced autoimmunity. Clin Exp Immunol 2002;127:12-19.

26 MacDonald AS, Straw AD, Dalton NM, Pearce EJ: Cutting edge: Th2 response induction by dendritic cells: a role for CD40. J Immunol 2002;168:537-540.
7 Yang X, HayGlass KT, Brunham RC: Genetically determined differences in IL-10 and IFNgamma responses correlate with clearance of Chlamydia trachomatis mouse pneumonitis infection. J Immunol 1996;156:4338-4344.

28 Yang X, Gartner J, Zhu L, Wang S, Brunham RC: IL-10 gene knockout mice show enhanced Th1-like protective immunity and absent granuloma formation following Chlamydia trachomatis lung infection. J Immunol 1999;162:1010-1017.

29 Njau F, Wittkop U, Rohde M, Haller H, Klos A, Wagner AD: In vitro neutralization of tumor necrosis factor-alpha during Chlamydia pneumoniae infection impairs dendritic cells maturation/function and increases chlamydial progeny. FEMS Immunol Med Microbiol 2009;55:215-225.
30 Penttila JM, Anttila M, Varkila K, Puolakkainen M, Sarvas M, Makela PH, Rautonen N: Depletion of CD8+ cells abolishes memory in acquired immunity against Chlamydia pneumoniae in BALB/c mice. Immunology 1999; 97:490-496.

31 Wizel B, Starcher BC, Samten B, Chroneos Z, Barnes PF, Dzuris J, Higashimoto Y, Appella E, Sette A: Multiple Chlamydia pneumoniae antigens prime $\mathrm{CD} 8+\mathrm{Tc} 1$ responses that inhibit intracellular growth of this vacuolar pathogen. J Immunol 2002;169:2524-2535. 